\title{
Capasso, Tromp Receive 1995 MRS Medals
}

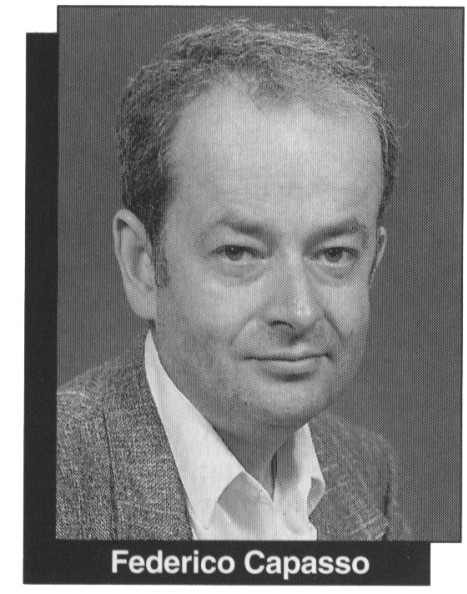

Two scientists from private research laboratories have been selected to receive the 1995 MRS Medal Awards, which recognize recent distinguished and innovative achievements or discoveries that are expected to have a major impact on the progress of any materials-related field. Federico Capasso (AT\&T Bell Laboratories) and Rudolf Tromp (IBM T.J. Watson Research Center) will receive their medals at the 1995 MRS Fall Meeting during the Awards Ceremony on Wednesday, November 29, at 6:00 p.m. in Salon E of the Boston Marriott Hotel.

\section{Federico Capasso}

Federico Capasso received the MRS Medal "for his seminal contributions to compositionally graded materials using bandgap engineering, and their innovative applications in electronics and optoelectronics." He has made innovative contributions to the design of compositionally graded materials grown by molecular beam epitaxy, described their unique transport and electro-optic properties, and pioneered their use in detectors and high-speed transistors.

Last year he and his team invented and demonstrated the quantum cascade laser, an accomplishment which was featured on the cover of Science. This laser uses a fundamentally new operating principle compared to conventional semiconductor lasers. It is based on electrons making radiative transitions between the discrete conduction band states of quantum wells. Charles Townes, who received the Nobel prize in physics for the invention of the laser, praised the quantum cascade laser in Science as "a beautiful piece of solid-state and laser physics," and called it a sharp departure from conventional laser design.

In another significant exploitation of graded-composition heterostructures, Capasso

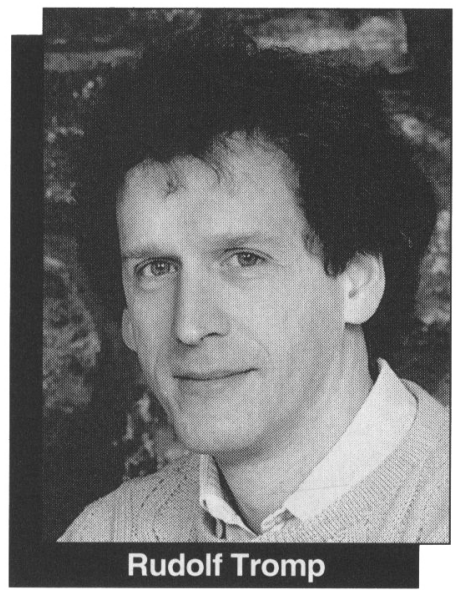

used multilayer materials with sawtooth composition profiles to experiment with staircase avalanche photodetector concepts. This led to a fundamental understanding of the reduction of multiplication noise in multilayer avalanche photodetectors.

Capasso received a $\mathrm{PhD}$ degree in physics from the University of Rome in 1973 and joined Fondazione Bordoni in Italy in 1974 as a research physicist. He began work as a visiting scientist at AT\&T Bell Laboratories in 1976 and joined the technical staff one year later. In 1987 he became head of the Quantum Phenomena and Device Research Department, a leadership role he continues to hold.

Capasso is a fellow of five scientific societies, is a member of the National Academy of Science and the National Academy of Engineering, and holds 21 U.S. and 43 non-U.S. patents. He is the recipient of the IEEE David Sarnoff Award, the Welker Memorial Gold Medal, and the Newcomb Cleveland Prize of the American Association for the Advancement of Science. He has published more than 175 papers and edited several books and a special issue of Physics Today.

Capasso will deliver his Medalist Award talk on Thursday, November 30, at 1:30 p.m. in Symposium CC: Spectroscopy of Heterojunctions. The title of his talk is "Bandgap Engineering of Compositionally Graded Semiconductors: Physics and Applications to Electronics and Photonics."

\section{Rudolf Tromp}

Rudolf Tromp received the MRS Medal "for his pioneering experiments on the role of atomic structure, surface stress, and surfactants in heteroepitaxial growth." He has made several major experimental contributions that elucidate the role of atomic structure, surface stress, and surface ener- gy in interface formation and growth. These new insights have culminated in the discovery and development of surfactantmediated epitaxial growth.

Tromp was the first to conduct a quantitative analysis of reconstructed silicon surface structures using detailed, full-scale Monte Carlo analysis of medium energy ion scattering experiments, a method that is now widely used. In the area of scanning tunneling microscopy and spectroscopy, Tromp and his co-workers were the first to obtain atom-resolved images of $\mathrm{Si}(001)$, settling the debate on dimer structure.

Probably the best known of Tromp's contributions to the understanding of interface formation and growth is the discovery and development of surfactant-mediated epitaxial growth. Materials that suffer from clustering and associated high defect densities when grown on clean surfaces can be grown as continuous two-dimensional films when an atomic "surfactant" layer is absorbed on the surface prior to growth, greatly improving the material quality. This method has been used successfully in semiconductor and metal systems alike:

Tromp developed a detailed understanding of the atomic origins of stress for $\mathrm{Si}$ and Ge surfaces and interfaces, which allowed new microscopic mechanisms of stress relief to be recognized and permitted a new epitaxy method to be developed. He has not only shown that surfactants work, but using median energy ion scattering, scanning tunneling microscopy, and low energy electron microscopy techniques, he has shown how they work.

Most recently, Tromp has studied the relaxation of misfit strain in heteroepitaxial growth by observing the nucleation, multiplication, and motion of misfit dislocations in real time as growth proceeds, inside the electron microscope.

Tromp received a physics degree from Technische Hogeschool Twente in The Netherlands in 1976. He pursued his thesis research at the FOM Institute for Atomic and Molecular Physics in Amsterdam, and received a $\mathrm{PhD}$ degree in physics from the University of Utrecht in 1982 . He joined the IBM T.J. Watson Research Center in 1983 and became manager of Interface Science in 1987, a position he continues to hold. He is a fellow of the American Physical Society and has published more than 115 papers.

Tromp will give his Medalist Award talk on Thursday, November 30, at 9:00 a.m. in Symposium J: In Situ Electron and Tunneling Microscopy of Dynamic Processes. The title of his talk is "Si(001) Homoepitaxial Growth." 


\section{As Simple As 1"-2"-3"}

Your Manufacturing Source for Small Diameter Silicon Wafers

Virginia Semiconductor, Inc.

1501 Powhatan Street, Fredericksburg, VA 22401 U.S.A.

Phone (540) 373-2900 Fax (540) 371-0371

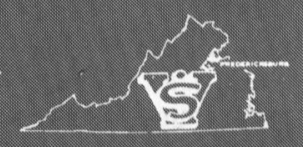

Circle No. 52 on Reader Service Card. 